\title{
LOS TRAYECTOS TRANSVERSALES DESDE LA MULTIDISCIPLINARIEDAD EN LOS ESTUDIOS CULTURALES
}

Inmaculada Gordillo (Universidad de Sevilla)

http://dx.doi.org/10.12795/AdMIRA.2009.01.08

Del feminismo a la teoría queer, del discurso poscolonial a los de la diáspora, del postestructuralismo a los enfoques multiculturales, de la crítica literaria a las teorías de la recepción y del consumo: los Estudios Culturales contemplan e interpretan los fenómenos sociales a partir de posiciones, perspectivas y visiones diversas $\mathrm{y}$ heterogéneas.

La diferenciación tajante de disciplinas y la separación entre humanidades, ciencias sociales, tecnológicas y otros campos científicos dejó de tener sentido cuando ciertas perspectivas transversales abarcaron ámbitos de estudio novedosos. En la segunda mitad del siglo XX algunos investigadores empezaron a observar la realidad desde enfoques interpretativos singulares que no se ceñían a una única disciplina. Precisamente ese fue el germen de los Cultural Studies. Y el crecimiento de las nuevas investigaciones ha dado lugar, en la actualidad, a multitud de publicaciones, departamentos universitarios, doctorados y facultades que se ocupan de ellos.

Sin pretender organizar un mapa histórico exhaustivo y diacrónico, existen una serie de precursores cuyas aportaciones organizaron los pilares de las investigaciones contemporáneas. El germen se sitúa en Reino Unido alrededor de 1956, aunque como señala Mattelart existen algunos precedentes en el siglo XIX relacionados con una tradición inglesa de pensamiento denominada Culture and Society y, en concreto, a los English Studies o estudios sobre literatura inglesa. Para autores como Thomas Carlyle (1795-1881), Mattew Arnold (1822-1888), William Morris (1834-1896) y, posteriormente, Frank Raymond Leváis (1895-1978), la literatura se transforma en el vínculo, símbolo y transmisor de la cultura. Todos ellos coinciden además en 
reflexionar sobre el papel de la cultura como instrumento de reconstitución de las naciones frente a las amenazas del Capitalismo.

Para Mattelart y Neveu (2004) no pueden dejar de citarse los trabajos de los denominados founding fathers de los estudios culturales: Richard Hoggart publica en 1957 una obra considerada fundamental, The Uses of Literacy: Aspects of WorkingClass Life with Special References to Publications and Entertainments, que arroja luces sobre la influencia de los medios de comunicación en la cultura de la clase obrera. En segundo término, los trabajos de inspiración marxista de Raymond Williams (como Culture and Society, 1958) y de Edward P. Thomson (The Making of the English Working Class, 1963), ambos provenientes del ámbito de la educación de adultos y vinculados más tarde a la New Left, que supondrían un interesante florecimiento de las ideas de izquierda en los sesenta. Los founding fathers dan cuenta de una visión de la historia a partir de las luchas sociales y como resistencia al orden impuesto por el sistema capitalista. Y precisamente Richard Hoggart funda en 1964 el CCCS (Centre for Contemporary Cultural Studies), cuyos componentes compondrán la Escuela de Birmingham, vinculada a estudios relacionados con las subculturas urbanas en relación a la etnicidad y el tiempo, el cine, la música, la literatura, el feminismo y la recepción de la cultura. El marxismo directo, las segundas lecturas de Marx o los vínculos del marxismo con el estructuralismo, el psicoanálisis o incluso la sociolingüística, constituyen algunas de las principales importaciones conceptuales del CCCS, un centro cuya principal función fue, durante mucho tiempo, la de legitimar los Estudios Culturales ante los estrictos y cerrados círculos académicos británicos. Además de los investigadores citados no podemos olvidar a Stuart Hall, el segundo de los directores del CCCS, y a diversos autores que publican en los Working Papers in Cultural Studies, la revista el centro.

Además, la escuela de Birmingham organizaría muchos de los conceptos que se utilizarán por posteriores estudios en torno a los bienes simbólicos. Nociones como la 
"ideología" que manifiestamente es una pieza del legado marxista, contribuyen a comprender como, en un contexto determinado, los sistemas de valores intervienen para promover resistencia o aceptación del statu quo. Así mismo, el concepto de "hegemonía" o construcción del poder con la conformidad de los dominados y el de "resistencia" o repertorio de obstáculos que ponen en marcha las clases populares para rebelarse y enfrentar esa dominación, terminan resultando esenciales. Y, por último, otra de las nociones básicas estaba constituida por la problemática de la "identidad" a través de la cual entran en juego variables como la generación, el género, la sexualidad o la etnicidad.

Por otro lado, el exilio de intelectuales integrantes de la escuela de Frankfurt a Norteamérica (Theodor Adorno, Herbert Marcuse y Max Horkheimer, sobre todo) y la obra de otros autores como Walter Benjamin influyeron notablemente en investigadores cuyo trabajo se desarrollará en Estados Unidos, como Paul Lazarsfeld, Bruno Bettelheim, Morris Janowitz y Frederic Jameson o Larry Grossberg. Desde posicionamientos más alejados del marxismo y con variables distintas a la escuela de Birmingham, los Cultural Studies norteamericanos rechazan la categoría de clase social como perspectiva para estudiar diferencias culturales, sustituyéndola por nociones como clase individual -alejada de grupos y procesos históricos- o análisis etnográfico.

Los Estudios Culturales en América Latina se ocupan de la producción simbólica de la realidad social latinoamericana desde dos vertientes bien diferenciadas. Por un lado están las preocupaciones de procesos recientes relacionados con la cultura de masas y los medios de comunicación, los procesos de consumo y la posmodernidad. En este ámbito destaca Néstor García Canclini, ocupado de la posmodernidad los lugares de intersección cultural o hibridaciones (culturas hegemónicas), y especialmente de la cultura popular en obras como Arte popular y sociedad en América Latina, (1977), La producción simbólica. Teoría y método en sociología del arte, (1979), Las culturas populares en el capitalismo, (1982), ¿De qué estamos hablando cuando hablamos de lo 
popular?, (1986) o Cultura transnacional y culturas populares (1988). Y también Jesús Martín Barbero, centrado en la investigación en torno a los medios de comunicación y el concepto de las mediaciones (en 1987 publica De los medios a las mediaciones). Esta noción se refiere a las formas, condiciones y espacios desde donde se construyen, producen y consumen los medios de comunicación. A partir de tres líneas esenciales construye sendos enfoques de mediación para investigar la producción cultural televisiva: en relación a los ritmos temporales, en la organización de la vida cotidiana, y en la correspondencia entre géneros narrativos e identidades culturales. A su vez, Martín Barbero se ocupa también del estudio de la posmodernidad como contexto de los productos culturales, de los procesos de la recepción, de las diferencias entre cultura popular y cultura de masas, de la multiculturalidad, etc. Destacan, entre otros, títulos como Globalización y multiculturalidad: notas para una agenda de investigación y Los ejercicios del ver. Hegemonía audiovisual y ficción televisiva, ambos de 1999.

En esta línea de trabajo sobresale a su vez Renato Ortiz, centrado en problemas de globalización y culturas locales, con obras como La conciencia fragmentada, Cultura brasileña e identidad nacional, A morte branca do feiticeiro negro; Cultura $y$ modernidad, Otro territorio, Modernidad y espacio, Japón y la modernidad-mundo, Lo próximo y lo distante.

Por otro lado, y a diferencia de los Cultural Studies europeos y norteamericanos, en Latinoamérica hay una línea de trabajo ajena a la cultura contemporánea, dedicada a temas de la primera mitad del XX, de todo el XIX e incluso de tiempos coloniales. Es una mirada hacia el pasado "para revisar las maneras en que nos hemos pensado antes para tratar de encontrar respuestas -o problematizaciones mayores- a los tiempos que hoy vivimos" (Ríos, 2002: 253). En esta línea destacan como precursores pensadores de la cultura como Simón Rodríguez, Andrés Bello, José Martí, Ángel Rama, Fernández Retamar, etc. 
La delimitación del campo epistemológico y la definición de los Estudios Culturales resulta uno de los principales escollos a la hora de abordar el tema. Las heterogéneas tendencias y enfoques, la diversidad metodológica y la procedencia desigual de los investigadores convierten el campo de la producción de los Estudios Culturales en un complejo corpus donde resulta fácil desubicarse.

Sin embargo, existe un punto de partida consensuado y común: el campo de los Estudios Culturales no constituye, en modo alguno, una disciplina ya que su origen se relaciona con "una forma de enfrentar los desafíos de una sociedad en continua transformación que no se deja 'leer' desde los marcos disciplinarios” (Reguillo, 2004: 2). La compartimentación del conocimiento queda obsoleta, por lo que el objeto de estudio, la metodología y las teorías dejan de poseer una delimitación precisa, situándose en ámbitos multidisciplinares e interdisciplinares. La variedad de temáticas y enfoques se caracteriza precisamente por la flexibilidad y la pluralidad.

La confluencia y la transversalidad se convierten en dos de los ejes que atraviesan el conjunto de la producción proveniente de los Estudios Culturales, por lo que los fenómenos sociales se contemplan desde distintas perspectivas, heterogéneas, diversas y complementarias. Aunque esas mismas perspectivas parten de un rechazo de los planteamientos de epistemologías monoculturales, orientándose claramente hacia posiciones multiculturales que consideran que la realidad es una construcción cuya existencia no es independiente de los actores que la interpretan, las teorías que le dan forma y el lenguaje que la conceptualiza. Se cuestiona entonces el racionalismo de la epistemología tradicional y se defiende la subjetividad de las interpretaciones del mundo.

La dimensión política y económica de los productos culturales contemporáneos organiza uno de los pilares básicos en la contemplación de las producciones simbólicas. Es importante la relación de éstas con el poder y con el contexto social o político. El entorno complejo de la cultura, las diversas formas en que se manifiesta y el contexto 
político y social constituyen parte esencial de los cimientos de los Estudios Culturales. Además, el componente de acción y compromiso se vincula a la crítica y valoración/evaluación de las prácticas culturales en las sociedades capitalistas industriales sobre todo. La influencia del marxismo y la visión del mismo de pensadores como Loius Althusser y Antonio Gramsci serán recurrentes entre los investigadores que organizan los pilares de estos campos interdisciplinares.

Con una sobresaliente capacidad de síntesis, Reguillo (2004: 3) organiza tres líneas claves dentro de los Estudios Culturales:

a) la importancia del sujeto y su relación con el marco político donde se desarrolla.

b) la necesidad de deconstruir configuraciones sociales históricamente consideradas como naturales mediante (procesos de exclusión, marginación y dominación).

c) la vinculación entre los elementos culturales y sus emisores (productores).

Un repaso a través de las temáticas atendidas por los Estudios Culturales obliga a citar las investigaciones de construcción social y discursiva del género, desde la crítica y la teoría feminista, con propuestas numerosas, plurales y desde posiciones heterogéneas. Pero los estudios que reivindican la igualdad de las mujeres en contra de las sociedades patriarcales dominantes no son los únicos campos de investigación en torno al género: los trabajos sobre la masculinidad, sobre la diversidad sexual atendiendo no solo a la homosexualidad, sino también a la bisexualidad y la transexualidad. Por otro lado, la teoría Queer rechaza las categorías tradicionales y las divisiones tajantes a partir del género, la biología o la orientación sexual e irrumpe con fuerza en las investigaciones de género en la última década del siglo XX. Las últimas aportaciones de Michel Foucault son decisivas en este ámbito: en su obra inconclusa Historia de la sexualidad analiza las relaciones entre poder, sexualidad y conocimiento. Foucault introduce los 
conceptos esenciales para la teoría queer como episteme, dispositivo o heteronormatividad. Además de Foucault, dentro de este ámbito de Estudios Culturales destacan autoras y autores como Judith Butler, Teresa de Lauretis, David Halperin, Martha Vicinius, Diana Fuss, Cindy Patton, Joseph Bristow, Gayle Rubin, Sue-Ellen Case, Ricardo Llamas, Simon Watney, Ed Cohen, Jonathan Dollimore, Lee Edelman, Ivonne Yarboro-Bejarano, Alan Sinfield, Eve Kosofsky Sedgwick o Leo Bersani.

La obra de Cliford Geertz La interpretación de las culturas influye notablemente en el principal objeto de investigación de los Estudios Culturales, sobre todo en el énfasis que promueve defendiendo la naturaleza simbólica de la cultura. Y precisamente, el concepto, la producción y las diversas manifestaciones de la cultura popular son temas recurrentes en los investigadores de los Cultural Studies, como Néstor García Canclini y otros muchos.

Los marcos conceptuales determinados por las relaciones entre sujetos sociales y sus prácticas culturales organizaron una distinción -ya clásica- entre cultura popular y alta cultura (o cultura de elite), relacionando la pertenencia a una clase social con una determina producción simbólica. A esta dualidad, con el crecimiento de los medios de comunicación, se añadió la cultura de masas, que con el desarrollo de los Estudios Culturales empezó a asimilarse al concepto de cultura popular. Así pues, se produjo una confusión amenazante del componente comunicativo: de la consideración del sujeto productor y difusor de elementos simbólicos populares se pasó a la masa consumidora de productos realizados independientemente de la intervención del sujeto social. Las industrias y políticas culturales adquieren entonces un rol trascendental dentro de las investigaciones de los Estudios Culturales.

Pero la fagocitación de la cultura popular por parte de la cultura mediática no es la única perspectiva de aproximación de los Estudios Culturales en relación este concepto. En 
torno a los años 60 y 70 del siglo XX destacó una tendencia que oponía la cultura popular a la que se enfrentaba a las manifestaciones culturales oficiales y hegemónicas. Como cultura de resistencia estaba encaminada a deslegitimar el orden simbólico vigente, a través de un compromiso de carácter político-social.

Y la última vertiente de aproximación a lo popular dentro del ámbito de la cultura viene dado por contenidos temáticos relacionado con elementos de folklore, de ritualización del pasado y mistificación del producto cultural, olvidando tanto el proceso social e industrial que lo rodea, así como los elementos que tiene relación con los sujetos productores o los procesos de recepción. Está vertiente también tiene cabida dentro de los Estudios Culturales, aunque el olvido del contexto político y social produce el rechazo de muchos investigadores.

A pesar de esta triple diferenciación del concepto de cultura popular, es una noción ligada a las reflexiones sobre la globalización del mundo contemporáneo, fenómenos que conlleva una mundialización/homogeneización de la cultura, así como la imposibilidad de estudiar sus productos a partir de diferenciaciones en compartimentos cerrados e incomunicados u olvidando a los medios de comunicación y las nuevas tecnologías.

Y estudiar la cultura obliga a ser conscientes del contexto en el que se produce. Por ello, el estudio de la Posmodernidad ha constituido uno de los lugares comunes de muchos de los investigadores del este ámbito epistemológico. Jameson, por ejemplo, defiende que es necesario "captar el posmodernismo no como un estilo, sino más bien como una pauta cultural: una concepción que permite la presencia y coexistencia de una gama de rasgos muy diferentes e incluso subordinados entre sí” (Jameson, 1984: 16). El posmodernismo es considerado un estilo de cultura que refleja un cambio de época en Occidente: hay una nueva forma de capitalismo, un giro hacia el efímero, descentralizado mundo de la tecnología, el consumismo y la industria cultural; las industrias de servicios, finanzas e información triunfan sobre las manufacturas 
tradicionales, y las políticas clásicas basadas en las clases ceden su lugar a una difusa serie de políticas de identidad (Eagleton, 1997: 11-12).

La globalización como surgimiento de tendencias hegemónicas de urbanización e industrialización de la cultura es una de las cuestiones recurrentes en las plurales investigaciones de los Estudios Culturales. Se relaciona con el paulatino borrado de las culturas nacionales como identidades individuales y, por tanto, con la alteración de los tradicionales paisajes sociales. Pero, como señala García Canclini (1995: 13), la globalización se entiende como "un proceso de fraccionamiento articulado del mundo y recomposición de sus pedazos. Con esto quiero afirmar que la globalización no es un simple proceso de homogeneización, sino de reordenamiento de las diferencias y desigualdades sin suprimirlas: por eso la multiculturalidad es un tema indisociable de los movimientos globalizadores". Así, es un concepto fuertemente relacionado con las políticas de identidad cultural, las problemáticas culturales referidas con los conceptos de raza, etnia y nación, los procesos asociados con la representación de la memoria histórica, el multiculturalismo, interculturalismo y el contexto cultural. Hay que tener en cuenta que a finales del siglo XX la enorme fuerza de las migraciones en el panorama internacional junto a los procesos contradictorios de homogenización y diferenciación que hacen mella en las representaciones de las culturas nacionales descubren un basto panorama para los Estudios Culturales. Por otro lado, no puede negarse la necesidad de afirmación de culturas locales, regionales o nacionales que se combinan con las globales, introduciendo el concepto de glocalización. A este panorama se le suman las aportaciones de las teorías orientalistas y poscoloniales, que se centran en las trayectorias que siguen las sociedades poscoloniales para construir su identidad nacional tras largas etapas de colonización. Las relaciones entre el imperialismo, el colonialismo, la figura del "otro" y las políticas identitarias han dado interesantes aportes a los estudios culturales, como los de Edward Saïd, Homi Bhabha, Fran Fanon, Duncan Ivision, Albert Memmi, etc. A partir de aquí se desarrollan diversas líneas de investigación como la del poscolonialismo de la hibridación o el feminismo poscolonial. 
Tras esta muestra de las temáticas, tendencias y enfoques habría que subrayar una serie de problemas presentes en el campo de los Estudios Culturales contemporáneos (García Canclini, 1997; Reguillo, 2004: 7-9). El primero de ellos denominado por García Canclini “estanflación”, es decir, estancamiento con inflación. La multiplicación de artículos, congresos, libros o debates no ofrece, sin embargo, descubrimientos, nuevas respuestas o aportaciones que supongan un verdadero avance en los Estudios Culturales. Los debates son efímeros e inconsistentes a causa de la rapidez y la abundante oferta.

Por otro lado, algunos intelectuales y académicos desconfían de los resultados de las investigaciones en Estudios Culturales debido a dos factores: la falta, por un lado, de una consistente reflexión teórica y epistemológica debido a la transdisciplinariedad y la cualidad del objeto de estudio, centrado en los procesos interculturales, así como la falta de estudios empíricos en algunos de los países donde los cultural studies arrancaron con fuerza -como en Estados Unidos- por otro. La tradicional división entre lo cuantitativo y lo cualitativo, entre el análisis del discurso frente a la estadística no puede contemplarse de forma simplificada. Es necesario un rigor multidimensional que organice los instrumentos metodológicos adecuados para cada momento.

Otro de los problemas viene dado por las llamadas "políticas de reconocimiento". Puesto que ninguna identidad es un fenómeno inmutable, analizar las diferencias y sus contextos sociales ofrece grandes dificultades, motivadas en parte por la multicausalidad y multicombinatoria de factores diversos.

La interacción entre globalidad y localidad ofrece articulaciones novedosas en relación a las prácticas culturales. El mundo contemporáneo, en continua transformación, se vuelve más complejo por las relaciones de complementariedad y oposición entre ambos planos. Existe entonces una tensión entre los diversos aspectos que abarcan los Estudios 
Culturales: el análisis de las estructuras, el contexto sociopolítico en relación a los discursos y a los procesos de recepción sin perder de vista al sujeto.

Otro de las zonas de peligro a la que se enfrentan los Estudios Culturales se relaciona con tendencias mesiánicas marcadas por verdades universales que conviertan las investigaciones en una especie de compendio moral o de nuevo testamento del pensamiento crítico. Para Reguillo, el objetivo sería abrirse al entendimiento de aquellos procesos, prácticas y productos que están marcando el avance de nuestras sociedades hacia un estadio más justo y democrático.

Para finalizar, habría que reflexionar en torno a la clave de los Estudios Culturales, marcados por la posición del sujeto en las investigaciones contemporáneas. Si el siglo XX supuso una verdadera invasión de una cientificidad en los campos tanto humanísticos como sociales, con la expulsión del sujeto de la historia, de la psicología, de la antropología y la sociología, sustituyéndolo por estímulos, comportamientos, respuestas, determinismos sociales y estructuras (Morín, 1994: 68), en la actualidad hay una restitución del sujeto. Aparece en las ciencias sociales y humanas como objeto de estudio y se toma consciencia de que la ciencia es una construcción de sujetos. Y con la aparición de los sujetos se empieza a señalar la pluralidad de concepciones de la realidad: "nunca hay una descripción correcta y verdadera de la realidad, sino muchas muy diferentes, según los criterios en los que se base el observador para la selección de sus informaciones, qué distinciones y valoraciones efectúa y desde qué perspectiva, con qué interés y con qué objetivos contempla su tema" (Simón, 1994: 133).

\section{Bibliografía}


BEVERLY, John, "Estudios culturales y vocación política", Revista de Crítica Cultural, núm. 12, Santiago de Chile, julio de 1996.

CASTRO-GÓMEZ, Santiago (2003): "Apogeo y decadencia de la teoría tradicional. Una visión desde los intersticios" en Walsh Catherine (editora), Estudios culturales latinoamericanos, Universidad Andina Simón Bolívar-Ediciones Abya-Yala, Quito.

CURRAN, James; MORLEY, David Y WALKERDINE, Valerie (compiladores) (1998): Estudios culturales y comunicación. Análisis, producción y consumo cultural de las políticas de identidad y el posmodernismo. Paidós Comunicación. Barcelona.

DUBE, Saurabh (1999): "Introducción: Temas e intersecciones de los pasados poscoloniales" en Saurabh Dube (ed.), Pasados poscoloniales. pp. 17-101. México, Colegio de México.

EAGLETON, Terry (1997): Las ilusiones del posmodernismo, Barcelona, Paidós.

ESCOBAR, Arturo (2003): "Mundos y conocimientos de otro modo: el programa de investigación de modernidad/colonialidad Latinoamericano" en Tabula Rasa, $\mathrm{n}^{\mathrm{o}}$ 1, pp. 51-86.

FOUCAULT, Michel (2005): Historia de la sexualidad (3 Vol.), Siglo XXI de España Editores, Madrid.

GARCÍA CANCLINI, Néstor (1982): "De lo primitivo a lo popular: interpretaciones de la desigualdad cultural" en Las culturas populares en el capitalismo. pp. 19-46. Habana: Casa de las Américas.

GARCÍA CANCLINI, Néstor (1995): Consumidores y ciudadanos: conflictos multiculturales de la globalización, Grijalbo, México.

GARCÍA CANCLINI, Néstor (coord.)(1996): Culturas en globalización, Caracas, Nueva Sociedad.

GARCÍA CNCLINI, Néstor (1997): "El malestar en lo estudios culturales" Fractal n 6, julio-septiembre año 2, volumen II, pp. 45-60. http://www.fractal.com.mx/F6cancli.html

GARCÍA-BEDOYA, Carlos (2001): "Los Estudios Culturales en debate: una mirada desde América Latina" en Revista De Critica Literaria Latinoamericana, Año XXVII, $\mathrm{N}^{\mathrm{o}}$ 54. Lima-Hanover, $2^{\circ}$. Semestre, pp. 195-211.

GROSFOGUEL, Ramón (2006): "La descolonización de la economía política y los estudios postcoloniales" en Tabula Rasa, n 4, pp. 17-48. 
GROSSBERG Lawrence (1997): "Cultural studies: What's in a Name? (One More Time)" en Bringing it all back home. Essays on Cultural Studies, Durham, Duke University Press.

GROSSBERG, Lawrence (2003): "Identidad y estudios culturales: ¿no hay nada más que eso?" en Stuart Hall y Paul du Gay (eds.), Cuestiones de Identidad, pp. 148-180. Buenos Aires: Amorrortu Editores.

GROSSBERG, Lawrence (2004): "Entre consenso y hegemonía. Notas sobre la forma hegemónica de la política moderna" en Tabula Rasa, n², pp. 49-57.

HALL, Stuart (1997): El trabajo de la representación. Instituto de Estudios Peruanos.

HALL, Stuart (1980): “Cultural Studies: Two Paradigms” en Media, Culture and Society.

HALL, Stuart (1985) "Signification, representation, ideology: Althusser and the poststructuralist debates" en Critical Studies in Mass Communication. 2: 91-114.

HALL, Stuart (1992): "Cultural Studies and Its Theoretical Legacies" en Lawrence Grossberg, Cary Nelson y Paula Treichler (eds.), pp.277-294. Cultural Studies. Routledge.

HALL, Stuart (2003): "Introducción: ¿Quién necesita la identidad?" en Stuart Hall y Paul du Gay (eds.), Cuestiones de Identidad. pp 13-39. Buenos Aires: Amorrortu Editores.

JAMESON, Fredric (1984): El posmodernismo o la lógica cultural del capitalismo avanzado, Barcelona, Paidós.

JAMESON, Fredric (1996): Teoría de la posmodernidad, Madrid, Trotta.

JAMESON, Fredric, (1992): El posmodernismo y lo visual, Valencia: Episteme.

JAMESON, Fredric (1993): "Conflictos interdisciplinarios en la investigación sobre cultura" en Alteridades, $\mathrm{n}^{\circ} 5$.

MALLON, Florencia (2001): "Promesa y dilema de los estudios subalternos. Perspectivas desde la historiografia latinoamericana" en Ileana Rodríguez (ed.), Convergencia de tiempos. Estudios sualternos / contextos latinoamericanos. Estado, cultura, subalternidad. pp. 117-154. Ámsterdam, Rodipi. 
MARTÍN-BARBERO, Jesús (1987). De los medios a las mediaciones. México: Gustavo Gili.

MARTÍN-BARBERO, Jesús (1999). Globalización y multiculturalidad: notas para una agenda de investigación.

MARTÍN-BARBERO, Jesús y REY, Germán (1999). Los ejercicios del ver. Hegemonía audiovisual y ficción televisiva. Barcelona: Editorial Gedisa.

MATO, Daniel (2002): "Estudios y otras prácticas latinoamericanas en cultura y poder" en Daniel Mato, (ed.), Estudios y otras prácticas latinoamericanas en cultura y poder. pp. 21-43. Caracas: Clacso.

MATTELART Armand y NEVEU Érik (2004): Introducción a los estudios culturales Barcelona, Paidós, 2004,

MATTELART, Armand (2006): Diversidad cultural y mundialización, Paidós, Barcelona.

MIGNOLO, Walter (2003): "Las humanidades y los estudios culturales: proyectos intelectuales y exigencias institucionales" en Catherine Walsh (ed.), Estudios culturales latinoamericanos. Retos desde y sobre la región andina. pp. 31-57. Quito: Abya-YalaUASB.

MILLER, Toby (2006): "What it is and what it isn't: Introducing... Cultural Studies" en Toby Miller (ed.), A Companion to Cultural Studies, pp. 1-21, Malden: Blackwell Publishing.

MORLEY, David (1998): "El postmodernismo: una guía básica" en J. Curran, D. Morley y V. Walkerdine (comps.), Estudios culturales y comunicación. pp. 85-83. Barcelona: Paidós.

NAÏR, Sami y DE LUCAS, Javier (1999): El desplazamiento del mundo: inmigración y temáticas de identidad, Ministerio de Asuntos Exteriores, Madrid.

REYNOSO, Carlos (2000): "Teorías y métodos" y "Conclusiones" en Apogeo y decadencia de los estudios culturales: una mirada antropológica. pp. 77-125, 301-311. Barcelona, Gedisa.

RICHARD, Nelly (1996): "Signos culturales y mediaciones académicas" en González, Beatriz, Cultura y tercer mundo, Nueva Sociedad, Caracas. 
RICHARD, Nelly (2001): "Globalización académica, estudios culturales y crítica latinoamericana" en Daniel Mato (ed.), Estudios Latinoamericanos sobre cultura y transformaciones sociales en tiempos de globalización. pp. 185-199. Buenos Aires, Clacso.

RÍOS, Alicia (2002): "Los Estudios Culturales y el estudio de la cultura en América Latina", en: Daniel Mato (coord.): Estudios y Otras Prácticas Intelectuales Latinoamericanas en Cultura y Poder. Caracas: Consejo Latinoamericano de Ciencias Sociales (CLACSO) y CEAP, FACES, Universidad Central de Venezuela, pp. 247-254.

SIMÓN, Fritz B. (1994). "Perspectiva interior y exterior. Cómo se puede utilizar el pensamiento sistémico en la vida cotidiana", en Watzlawick, Paul y Krieg, Peter (Ed.), El ojo del observador. pp. 132-142, Barcelona, Gedisa.

WILLIAMS, Raymond (1997): "El futuro de estudios culturales" en La politica del modernismo. Contra los nuevos conformistas, pp. 187-200, Buenos Aires, Manantial.

YÚDICE, G (1993): "Tradiciones comparativas de estudios culturales: América Latina y Estados Unidos" en Alteridades, $\mathrm{n}^{\mathrm{0}} 5$. 